\title{
Shearing and geodesic axially symmetric perfect fluids that do not produce gravitational radiation
}

\author{
L. Herrerd: \\ Escuela de Física, Facultad de Ciencias, Universidad Central de Venezuela, Caracas 1050, \\ Venezuela and Instituto Universitario de Física Fundamental y Matemáticas, \\ Universidad de Salamanca, Salamanca 3700\%, Spain \\ A. Di Priscd \\ Escuela de Física, Facultad de Ciencias, Universidad Central de Venezuela, Caracas 1050, Venezuela \\ J. Ospind团 \\ Departamento de Matemática Aplicada and Instituto Universitario de Física Fundamental y Matemáticas, \\ Universidad de Salamanca, Salamanca 3700\%, Spain

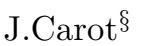 \\ Departament de Física, Universitat Illes Balears, E-07122 Palma de Mallorca, Spain
}

(Dated: August 7, 2018)

\begin{abstract}
Using a framework based on the $1+3$ formalism we carry out a study on axially and reflection symmetric perfect and geodesic fluids, looking for possible models of sources radiating gravitational waves. Therefore, the fluid should be necessarily shearing, for otherwise the magnetic part of the Weyl tensor vanishes, leading to a vanishing of the super-Poynting vector. However, for the family of perfect, geodesic fluids considered here, it appears that all possible cases reduce to conformally flat, shear-free, vorticity-free, fluids, i.e Friedmann-Roberston-Walker. The super-Poynting vector vanishes and therefore no gravitational radiation is expected to be produced. The physical meaning of the obtained result is discussed.
\end{abstract}

PACS numbers: 04.40.-b, 04.40.Nr, 04.40.Dg

Keywords: Relativistic Fluids, nonspherical sources, interior solutions.

\section{INTRODUCTION}

In a recent paper [1] the $1+3$ formalism [2-5] has been used to develop a general framework for studying axially symmetric dissipative fluids. Besides some results exhibited in [1], the above mentioned framework has been applied to the shear-free case [6]. As the result of such a study, it follows that all geodesic and shear-free fluids, are irrotational, and as consequence of this, also purely electric. Such a result holds for a general fluid (not necessarily perfect).

Now, if we define a state of intrinsic gravitational radiation (at any given point), to be one in which the superPoynting vector does not vanish for any unit timelike vector [7 9], then since the vanishing of the magnetic part of the Weyl tensor implies the vanishing of the superPoynting vector, it is clear that when looking for gravitationally radiating sources (at least under the geodesic condition) we should consider shearing fluids. It is worth recalling that the tight link between the super-Poynting vector and the existence of a state of radiation is firmly supported by the relationship between the former and

\footnotetext{
* lherrera@usal.es

† alicia.diprisco@ciens.ucv.ve

$\ddagger$ j.ospino@usal.es

$\S$ jcarot@uib.cat
}

the Bondi news function [10] (see [11] for a discussion on this point).

From the comments above, and as a further step towards the understanding of gravitationally radiating sources, we shall consider in this work the simplest fluid distribution which we might believe to be compatible with a non-vanishing super-Poynting vector, namely: perfect fluid under the geodesic condition.

However, our investigation shows that all possible models, sourced by a perfect fluid (which of course includes the pure dust configuration as a particular subcase), belonging to the family of the line element considered here, do not radiate gravitational waves during their evolution.

We shall discuss about this result and its relationship with the fact that the process of radiation (including absorption and/or Sommerfeld type conditions) is not a reversible one.

We shall heavily rely on the general framework developed in [1], keeping the same notation, and just reducing the general equations to the particular case considered here. These will be presented in an Appendix.

\section{THE PERFECT, GEODESIC, FLUID}

We shall consider axially and reflection symmetric perfect fluid distributions (not necessarily bounded). For 
such a system, we assume that the line element may be written in "Weyl spherical coordinates", as:

$$
d s^{2}=-A^{2} d t^{2}+B^{2}\left(d r^{2}+r^{2} d \theta^{2}\right)+C^{2} d \phi^{2}+2 G d \theta d t
$$

where $A, B, C, G$ are positive functions of $t, r$ and $\theta$. We number the coordinates $x^{0}=t, x^{1}=r, x^{2}=\theta, x^{3}=\phi$.

The specific form of the line (1) deserves some comments. Our original goal, here as well as in [1] and [6], has been to describe the gravitational radiation process, through the physical properties of its source. Such an endeavour, should, eventually, lead to the obtention of a specific source of gravitational radiation. With this purpose in mind, and in order to render the problem under consideration, analytically handable, we have imposed the highest degree of symmetry compatible with the existence of gravitational radiation. For vacuum, it is represented by the Bondi metric [10]. Here, following the framework developed in [1], we have restricted the line element as much as possible, always allowing for the existence of gravitational radiation (at least for the most general matter distribution). As the result of these restrictions, we are able to manipulate the resulting expressions, by purely analytical procedures. However, this is obtained at the price of dealing with a space-time, that is not the more general one, compatible with axial symmetry (see the discussion below (50) ).

The energy momentum tensor in the "canonical" form reads:

$$
T_{\alpha \beta}=(\mu+P) V_{\alpha} V_{\beta}+P g_{\alpha \beta},
$$

where as usual, $\mu, P$ and $V_{\beta}$ denote the energy density, the isotropic pressure, and the four velocity, respectively. As in [1] we are assuming the fluid to be comoving in the coordinates of (1).

The shear tensor is defined by two scalar functions $\sigma_{I}, \sigma_{I I}$, which in terms of the metric functions read (see eqs. $(20-25)$ in [1]):

$$
\begin{aligned}
2 \sigma_{I}+\sigma_{I I}= & \frac{3}{A}\left(\frac{\dot{B}}{B}-\frac{\dot{C}}{C}\right) \\
2 \sigma_{I I}+\sigma_{I}= & \frac{3}{A^{2} B^{2} r^{2}+G^{2}}\left[A B^{2} r^{2}\left(\frac{\dot{B}}{B}-\frac{\dot{C}}{C}\right)\right. \\
& \left.+\frac{G^{2}}{A}\left(-\frac{\dot{A}}{A}+\frac{\dot{G}}{G}-\frac{\dot{C}}{C}\right)\right]
\end{aligned}
$$

where dots denote derivatives with respect to $t$.

For the other kinematical variables (the expansion, the four acceleration and the vorticity) we have:

the expansion

$$
\begin{aligned}
\Theta= & V_{; \alpha}^{\alpha} \\
= & \frac{A B^{2}}{r^{2} A^{2} B^{2}+G^{2}}\left[r^{2}\left(2 \frac{\dot{B}}{B}+\frac{\dot{C}}{C}\right)\right. \\
& \left.+\frac{G^{2}}{A^{2} B^{2}}\left(\frac{\dot{B}}{B}-\frac{\dot{A}}{A}+\frac{\dot{G}}{G}+\frac{\dot{C}}{C}\right)\right] .
\end{aligned}
$$

The four acceleration

$$
a_{\alpha}=V^{\beta} V_{\alpha ; \beta}=a_{I} K_{\alpha}+a_{I I} L_{\alpha}
$$

with vectors $\mathbf{K}$ and $\mathbf{L}$ having components:

$$
K_{\alpha}=(0, B, 0,0) ; \quad L_{\alpha}=\left(0,0, \frac{\sqrt{A^{2} B^{2} r^{2}+G^{2}}}{A}, 0\right),
$$

and where the two scalar functions $\left(a_{I}, a_{I I}\right)$ are defined by (see eq.(17) in [1])

$$
\begin{aligned}
a_{I} & =\frac{A^{\prime}}{A B}, \\
a_{I I} & =\frac{A}{\sqrt{A^{2} B^{2} r^{2}+G^{2}}}\left[\frac{G}{A^{2}}\left(-\frac{\dot{A}}{A}+\frac{\dot{G}}{G}\right)+\frac{A_{, \theta}}{A}\right],
\end{aligned}
$$

whereas the vorticity vector is defined through a single scalar $\Omega$, given by (see eq.(29) in [1])

$$
\Omega=\frac{\left(A G^{\prime}-2 G A^{\prime}\right)}{2 A B \sqrt{A^{2} B^{2} r^{2}+G^{2}}}
$$

where primes denote derivatives with respect to $r$.

We shall restrict our system to the case of vanishing fouracceleration $a_{\alpha}=0$. This condition implies that

$$
a_{I}=\frac{A^{\prime}}{A B}=0 \Rightarrow A=\tilde{A}(t, \theta)
$$

and

$$
a_{I I}=0 \Rightarrow \frac{G}{A^{2}}\left(-\frac{\dot{A}}{A}+\frac{\dot{G}}{G}\right)+\frac{A, \theta}{A}=0
$$

or

$$
\left(\frac{G}{A}\right)=-A_{, \theta} \Rightarrow \frac{G}{A}=-\int A_{, \theta} d t+\tilde{G}(r, \theta) .
$$

Given that $G(t, 0, \theta)=0$, from (13) we find that

$$
A_{, \theta}=0 \Rightarrow A=\tilde{A}(t) \text { and } G=\tilde{G} \tilde{A}
$$

In this case, reparametrizing the time coordinate, the line element takes the form

$$
d s^{2}=-d t^{2}+B^{2}\left(d r^{2}+r^{2} d \theta^{2}\right)+2 \tilde{G}(r, \theta) d t d \theta+C^{2} d \phi^{2},
$$


and the kinematical quantities become

$$
\begin{gathered}
\Theta=\frac{2 B^{2} r^{2}+\tilde{G}^{2}}{B^{2} r^{2}+\tilde{G}^{2}} \frac{\dot{B}}{B}+\frac{\dot{C}}{C}, \\
\Omega=\frac{\tilde{G}^{\prime}}{2 B \sqrt{B^{2} r^{2}+\tilde{G}^{2}}}, \\
\sigma_{I}=\frac{B^{2} r^{2}+2 \tilde{G}^{2}}{B^{2} r^{2}+\tilde{G}^{2}} \frac{\dot{B}}{B}-\frac{\dot{C}}{C}, \\
\sigma_{I}-\sigma_{I I}=\frac{3 \tilde{G}^{2}}{B^{2} r^{2}+\tilde{G}^{2}} \frac{\dot{B}}{B} .
\end{gathered}
$$

Now, from the regularity conditions, necessary to ensure elementary flatness in the vicinity of the axis of symmetry, and in particular at the center (see [12], 13], 14]), we should require that as $r \approx 0$

$$
\Omega=\sum_{n \geq 1} \Omega^{(n)}(t, \theta) r^{n}
$$

implying, because of (17) that in the neighborhood of the center

$$
\tilde{G}=\sum_{n \geq 3} \tilde{G}^{(n)}(\theta) r^{n}
$$

This last result in turn implies that as $r$ approaches 0 ,

$$
\sigma_{I}-\sigma_{I I}=\sum_{n \geq 4}\left[\sigma_{I}^{(n)}(t, \theta)-\sigma_{I I}^{(n)}(t, \theta)\right] r^{n} .
$$

Now, for the length of an orbit at $t, \theta$ constant, to be $2 \pi r$, close to the origin (elementary flatness), we may write, as $r \rightarrow 0$,

$$
C \approx r \gamma(t, \theta)
$$

implying

$$
C^{\prime} \approx \gamma(t, \theta), \quad C_{, \theta} \approx r \gamma_{, \theta}
$$

where $\gamma(t, \theta)$ is an arbitrary function of its arguments, which as appears evident from the elementary flatness condition, cannot vanish anywhere within the fluid distribution.

Finally, observe that a combination of (16)-(19) produces

$$
\left(\sigma_{I}-\sigma_{I I}\right)\left(B r^{2}+\tilde{G}^{2}\right)=\tilde{G}^{2}\left(\Theta+\sigma_{I}\right)
$$

We shall next make use of the full set of equations deployed in 1], written for the specific case considered here, they are given in the Appendix A.
Thus, taking the time derivative of the above equation and combining with (A1), (A2) and (A4), we obtain

$\mathcal{E}_{I I}-\mathcal{E}_{I}=\frac{\sigma_{I}-\sigma_{I I}}{\Theta+\sigma_{I}}\left[3 \Omega^{2}-\mathcal{E}_{I}-Y_{T}-\frac{1}{3}\left(\Theta+\sigma_{I}\right)\left(\Theta+\sigma_{I I}\right)\right]$,

and

$\mathcal{E}_{I I}-\mathcal{E}_{I}=\frac{\sigma_{I}-\sigma_{I I}}{\Theta+\sigma_{I I}}\left[3 \Omega^{2}-\mathcal{E}_{I I}-Y_{T}-\frac{1}{3}\left(\Theta+\sigma_{I}\right)\left(\Theta+\sigma_{I I}\right)\right]$,

where (5) has also been used.

In the above expressions, $\mathcal{E}_{I, I I}$, are two of the three scalar functions defining the electric part of the Weyl tensor (see [1] for details) (the third scalar function is denoted $\left.\mathcal{E}_{K L}\right)$. Also, $Y_{T}$, is one of the structure scalars obtained from the orthogonal splitting of the Riemann tensor which are defined in eqs.(38-50) in [1].The others are denoted by $Y_{I, I I, K L}, X_{T, I, I I, K L}, Z_{I, I I, I I I, I V}$.

From the above equations and (21) it follows at once that as $r \approx 0$

$$
\mathcal{E}_{I}-\mathcal{E}_{I I}=\sum_{n \geq 4}\left[\mathcal{E}_{I}^{(n)}(t, \theta)-\mathcal{E}_{I I}^{(n)}(t, \theta)\right] r^{n},
$$

and (22), unless $\Theta+\sigma_{I}=\Theta+\sigma_{I I}=0$.

However, this last condition cannot be satisfied. Indeed the absence of singularities in $H_{2}$ at $r=0$ requires from (A9), that $\sigma_{I, I I} \approx r$ as $r \approx 0$ which would produce $\Theta \approx 0$, implying in turn because of A1

$$
Y_{T}(r=0)=(\mu+3 P)_{r=0}=0 .
$$

Now, in order to satisfy (29) we have to assume, the equation of state

$$
(\mu+3 P)=0,
$$

at least in the neighborhood of the center. Excluding this possibility on physical grounds, we have to assume $\Theta+\sigma_{I, I I} \neq 0$.

Next, from the combination of A10, A12 and (A13) we obtain

$$
\frac{\tilde{G}}{\sqrt{B^{2} r^{2}+\tilde{G}^{2}}}\left[\frac{H_{2} \tilde{G}^{\prime}}{2 B \sqrt{B^{2} r^{2}+\tilde{G}^{2}}}+H_{1}\left(\frac{\dot{B}}{B}-\frac{\dot{C}}{C}\right)\right]=0
$$

where $H_{1}, H_{2}$ are the two scalar functions defining the magnetic part of the Weyl tensor.

From the above equation it follows that either $\tilde{G}=$ $0 \Rightarrow \Omega=0$, or

$$
H_{2} \Omega+H_{1}\left[\frac{2 \sigma_{I}+\sigma_{I I}}{3}\right]=0,
$$

where (17)- (19) have been used.

Since $\Omega$ goes to zero at the center, as $r$, we are left with three possibilities to proceed further, namely:

1. The vorticity is assumed to vanish $(\tilde{G}=0)$. 
2. $\tilde{G} \neq 0$ and the term within the square bracket in (32) does not vanish at the center, meaning that we may write

$$
\sigma_{I}=\sum_{n=0} \sigma_{I}^{(n)} r^{n}, \quad \sigma_{I I}=\sum_{n=0} \sigma_{I I}^{(n)} r^{n} .
$$

3. $\tilde{G} \neq 0$ and the term within the square bracket in (32) does vanish at the center implying that

$$
\sigma_{I}=\sum_{n \geq 1} \sigma_{I}^{(n)} r^{n}, \quad \sigma_{I I}=\sum_{n \geq 1} \sigma_{I I}^{(n)} r^{n} .
$$

Next, contracting (A.7) in [1] with $\mathbf{K}$ and $\mathbf{L}$ we obtain, respectively

$$
\begin{gathered}
P^{\prime}=0 \Rightarrow P=P(t, \theta), \\
\tilde{G} P_{, t}+P_{, \theta}=0,
\end{gathered}
$$

which, due to the regularity conditions on the axis of symmetry, implies that either $\tilde{G}=0$ and $P=P(t)$, or $\tilde{G} \neq 0$ and $P=$ constant.

We shall now analyze the three possible cases mentioned before.

\section{A. $\tilde{G}=0$}

From the well established link between radiation and vorticity (see 15 and references therein), it might be inferred that no gravitational radiation is expected to be emitted in this case.

In what follows we shall provide a formal proof of this result.

As mentioned before, in this case we have $P=P(t)$; then, from (16), (3) and (4) we get

$$
\Theta=2 \frac{\dot{B}}{B}+\frac{\dot{C}}{C}, \sigma_{I}=\sigma_{I I}=\tilde{\sigma}=\frac{\dot{B}}{B}-\frac{\dot{C}}{C},
$$

implying:

$$
\Theta=2 \tilde{\sigma}+\frac{3 \dot{C}}{C}=\frac{3 \dot{B}}{B}-\tilde{\sigma} .
$$

Next, equation (A1) is the Raychaudhury equation for this case, which reads

$$
\dot{\Theta}+\frac{1}{3} \Theta^{2}+\frac{2}{3} \tilde{\sigma}^{2}+Y_{T}=0
$$

or, using (38)

$$
\dot{\tilde{\sigma}}-\frac{1}{3} \tilde{\sigma}^{2}+\frac{2}{3} \Theta \tilde{\sigma}+\frac{3}{2} \frac{\ddot{C}}{C}+\frac{Y_{T}}{2}=0,
$$

whereas from (A2)-

$$
\begin{gathered}
\dot{\tilde{\sigma}}-\frac{1}{3} \tilde{\sigma}^{2}+\frac{2}{3} \Theta \tilde{\sigma}+Y_{I}=0, \\
Y_{K L}=0 \Rightarrow X_{K L}=0,
\end{gathered}
$$

and

$$
\dot{\tilde{\sigma}}-\frac{1}{3} \tilde{\sigma}^{2}+\frac{2}{3} \Theta \tilde{\sigma}+Y_{I I}=0 .
$$

Then, from (40)-43) we get

$$
Y_{I}=Y_{I I}=Y=\frac{3}{2} \frac{\ddot{C}}{C}+\frac{Y_{T}}{2},
$$

and using (42)-(45) and (47)-(50) in [1] it follows that

$$
\begin{aligned}
X_{I} & =X_{I I}=X, \mathcal{E}_{I}=\mathcal{E}_{I I}=\mathcal{E}, Y=-X=\mathcal{E}, \\
\mathcal{E}_{K L} & =X_{K L}=Y_{K L}=0 .
\end{aligned}
$$

Two constraint equations follow from (A6) and (A7)

$$
2 \Theta^{\prime}-\tilde{\sigma}^{\prime}-3 \tilde{\sigma} \frac{C^{\prime}}{C}=0,
$$

$$
2 \Theta_{, \theta}-\tilde{\sigma}_{, \theta}-3 \tilde{\sigma} \frac{C_{, \theta}}{C}=0,
$$

whereas from (A8) and (A9) we get

$$
\begin{gathered}
H_{1}=-\frac{\tilde{\sigma}}{2 B r}\left(\frac{C_{, \theta}}{C}+\frac{\tilde{\sigma}_{, \theta}}{\tilde{\sigma}}\right)=-\frac{(\tilde{\sigma} C)_{, \theta}}{2 B r C}, \\
H_{2}=\frac{\tilde{\sigma}}{2 B}\left(\frac{\tilde{\sigma}^{\prime}}{\tilde{\sigma}}+\frac{C^{\prime}}{C}\right)=\frac{(\tilde{\sigma} C)^{\prime}}{2 B C} .
\end{gathered}
$$

From the two equations above we find that if the magnetic part of the Weyl tensor vanishes, then

$$
\tilde{\sigma} C=\psi(t)
$$

where $\psi(t)$ is an arbitrary integration function. The above equation implies, because of the regularity condition $C(t, 0, \theta)=0$, that $\tilde{\sigma}=0$.

This last result in turn implies (as mentioned before) that our line element (11) (or (15)) is not the more general one, since in the spherically symmetric limit, it does not contain the Lemaitre-Tolman-Bondi metric. Also, the Szekeres metric [16 19] (its axially symmetric version) cannot be recovered from (15). In other words, the shear of the fluid (in our models) is sourced by the magnetic part of the Weyl tensor. Therefore in the spherically symmetric limit, we recover the shear-free case.

Another important conclusion emerges from (48), (49). Indeed, since $B$ is regular at the origin $(r \approx 0)$, whereas 
$C$ behaves as $C \approx r$, then for the magnetic Weyl tensor to be regular at the origin, we must demand there $\tilde{\sigma} \approx r$ (at least). But then, (41) or (43) implies that $\mathcal{E} \approx r$ at the origin.

Also, from (47), it follows that $\Theta_{, \theta} \approx 0$ (in the neighborhood of the center), and then from (38) it follows that

$$
\frac{\gamma, \theta}{\gamma}=l(\theta)
$$

where $l(\theta)$ is an arbitrary function of its argument.

Next, using (46) and (47) we may write

$$
\begin{gathered}
2(\Theta+\sigma)^{\prime}=\frac{(3 \tilde{\sigma} C)^{\prime}}{C} \Rightarrow 2\left(\frac{\dot{B}}{B}\right)^{\prime}=\frac{(\tilde{\sigma} C)^{\prime}}{C}, \\
2(\Theta+\sigma)_{, \theta}=\frac{(3 \tilde{\sigma} C)_{, \theta}}{C} \Rightarrow 2\left(\frac{\dot{B}}{B}\right)_{, \theta}=\frac{(\tilde{\sigma} C)_{, \theta}}{C} .
\end{gathered}
$$

Feeding back the two equations above into (48) and (49) we obtain

$$
B r H_{1}=-\left(\frac{\dot{B}}{B}\right)_{, \theta}, B H_{2}=\left(\frac{\dot{B}}{B}\right)^{\prime}
$$

On the other hand (A11) and (A16), produce, respectively

$H_{2, \theta}+H_{2}\left(\frac{2 C_{, \theta}}{C}-\frac{B, \theta}{B}\right)=r\left[H_{1}^{\prime}+H_{1}\left(\frac{2 C^{\prime}}{C}-\frac{(B r)^{\prime}}{B r}\right)\right]$,

and

$$
\left(H_{1} B r C^{2}\right)^{\prime}+\left(H_{2} B C^{2}\right)_{, \theta}=0
$$

whereas the combination of the equations above with (48) and (49) produces

$$
H_{1} C^{\prime} r+H_{2} C_{, \theta}=0
$$

or

$$
-\left(\frac{\dot{B}}{B}\right)_{, \theta} C^{\prime}+\left(\frac{\dot{B}}{B}\right)^{\prime} C_{, \theta}=0
$$

Next, using (37) in (52) and (53), it follows that

$$
\begin{gathered}
\left(\frac{\dot{B}}{B}\right)_{, \theta}=\frac{\dot{B} C_{, \theta}}{B C}-\frac{\dot{C}_{, \theta}}{C} \\
\left(\frac{\dot{B}}{B}\right)^{\prime}=\frac{\dot{B} C^{\prime}}{B C}-\frac{\dot{C}^{\prime}}{C}
\end{gathered}
$$

Now, combining the two equations above with (58) we may write

$$
\frac{\dot{C}_{, \theta}}{C_{, \theta}}=\frac{\dot{C}^{\prime}}{C^{\prime}} \Rightarrow C^{\prime}=\gamma(r, \theta) C_{, \theta}
$$

implying

$$
C=\kappa(t) \tilde{C}(r, \theta),
$$

and, because of (57)

$$
H_{2}=-r \gamma(r, \theta) H_{1} .
$$

Then, using (61) in (58) we get

$$
\frac{B^{\prime}}{B}=\frac{B_{, \theta}}{B} \gamma(r, \theta)+\epsilon(r, \theta)
$$

with

$$
\epsilon=\frac{\gamma_{, \theta} r+\gamma \gamma^{\prime} r^{3}-1}{r\left(1+r^{2} \gamma^{2}\right)}
$$

implying

$$
B=T(t) \tilde{B}(r, \theta) \Rightarrow \frac{\dot{B}}{B}=\frac{\dot{T}}{T}=f(t),
$$

where $T(t)$ and $\tilde{B}(r, \theta)$ are arbitrary functions of their arguments.

Using the above result in (54), it follows at once that $H_{1}=H_{2}=0$, which as mentioned before implies $\tilde{\sigma}=$ 0 . This last result in turn implies that the expansion scalar only depends on $t$ and taking into account that $Y_{T}=4 \pi(\mu+P)$, the Raychaudhury equation requires $\mu=\mu(t)$.

Next, A14 reads in this case

$$
\frac{1}{3} \mathcal{E}^{\prime}+\frac{C^{\prime}}{C} \mathcal{E}=0
$$

which due to the fact that $\mathcal{E} \approx 0$ at the origin, implies $\mathcal{E}=0$.

Thus our spacetime is conformally flat, shear-free, and due to the fact that the fluid is perfect, it is a FRW spacetime, in agreement with the result obtained in 6 .

For the sake of completeness we shall sketch another proof of the above result in the Appendix B.

Now, from the fact that our system is conformally flat it appears that it does not radiate gravitationally (according to the criterium commented in the Introduction).

Indeed, the super-Poynting vector can be written (ec. (55) in [1]) as

$$
P_{\alpha}=P_{I} K_{\alpha}+P_{I I} L_{\alpha},
$$

where according to (56) in [1], and (45), we have for the two scalars defining the super-Poynting vector

$$
P_{I}=2 H_{2} Y ; \quad P_{I I}=-2 H_{1} Y .
$$

Thus, the vanishing of $\mathcal{E}, H_{1}$ and $H_{2}$, as in our case, implies the vanishing of gravitational radiation. 
B. $\tilde{G} \neq 0$ and the term within the square bracket in (32) does not vanish at the center.

In this case, from the regularity of $\mathrm{H}_{2}$ at the center we may write, in the neighborhood of $r \approx 0$,

$$
H_{2}=\sum_{n=0} H_{2}^{(n)} r^{n}
$$

in which case, (32) implies

$$
H_{1}=\sum_{n \geq 1} H_{1}^{(n)} r^{n} .
$$

Replacing the two above expressions in a combination of (A10) and (A12), and using (21), (A3), (28), (22) we find that the lowest order of $r$ in $H_{1}$ and $H_{2}$ is raised as

$$
H_{1}=\sum_{n \geq 6} H_{1}^{(n)} r^{n}, \quad H_{2}=\sum_{n \geq 5} H_{2}^{(n)} r^{n} .
$$

Next, from (A14) we may write (close to the center)

$$
\mathcal{E}_{I}=\sum_{n \geq 1} \mathcal{E}_{I}^{(n)} r^{n}, \quad \mathcal{E}_{I I}=\sum_{n \geq 1} \mathcal{E}_{I I}^{(n)} r^{n},
$$

for otherwise there would be an inadmissible singularity in the $r$-derivative of the energy density at the origin.

Feeding back the two expressions above in a combination of (A12) and (A13), it follows from the lowest order in $r$, that in the neighborhood of the center

$$
(\mu+P) \sigma_{I} \approx O(r)
$$

which implies that,

$$
(\mu+P) \approx O(r)
$$

which of course is impossible unless we assume, close to the center, the equation of state

$$
(\mu+P)=0
$$

Excluding this possible situation from physical considerations, we have to require that

$$
\sigma_{I}=\sum_{n \geq 1} \sigma_{I}^{(n)} r^{n}
$$

which in turn implies

$$
\sigma_{I I}=\sum_{n \geq 1} \sigma_{I I}^{(n)} r^{n}
$$

because of (22).

But of course this contradicts the main assumption of this case about the nonvanishing of the term within the square bracket in (32), (Eq. (33)).

Thus we have to assume (34), implying that the term within the square bracket in (32) vanishes at the center as $r$.

\section{C. $\tilde{G} \neq 0$, and the term within the square bracket in (32) does vanish at the center.}

Then, it follows at once from (16) and (18), close to the center, that

$$
\frac{\dot{B}}{B} \approx \frac{\dot{C}}{C}, \quad \Theta \approx \frac{3 \dot{C}}{C} \approx \frac{3 \dot{B}}{B} .
$$

Next, from the lowest order of $r$ in (A2) and (A4) it appears that

$$
\mathcal{E}_{1}=\sum_{n \geq 1} \mathcal{E}_{I}^{(n)} r^{n}, \quad \mathcal{E}_{I I}=\sum_{n \geq 1} \mathcal{E}_{I I}^{(n)} r^{n}
$$

and, (19), (2A3), (26) and (27), as $r \approx 0$, produce (22), (28) and

$$
\mathcal{E}_{K L}=\sum_{n \geq 5} \mathcal{E}_{K L}^{(n)} r^{n}
$$

Excluding singularities of the scalars $H_{1}, H_{2}$, at the origin, we may write:

$$
H_{1}=\sum_{n=0} H_{1}^{(n)} r^{n}, \quad H_{2}=\sum_{n=0} H_{2}^{(n)} r^{n},
$$

Then, looking for the lowest order of $r$ in A10 A13 and A16) we obtain respectively:

$$
H_{1, \theta}^{(0)}=-H_{1}^{(0)} \frac{\gamma_{, \theta}}{\gamma}
$$

$$
H_{2, \theta}^{(0)}+H_{2}^{(0)}\left(\frac{2 \gamma, \theta}{\gamma}-\frac{B, \theta}{B}\right)-H_{1}^{(0)}=0
$$

$$
H_{2}^{(0)}=-H_{1}^{(0)}\left(\frac{\gamma, \theta}{\gamma}-\frac{B, \theta}{B}\right)
$$

$$
H_{1, \theta}^{(0)}+H_{1}^{(0)} \frac{B, \theta}{B}-H_{2}^{(0)}=0
$$

$$
3 H_{1}^{(0)}+H_{2, \theta}^{(0)}+H_{2}^{(0)}\left(\frac{2 \gamma, \theta}{\gamma}+\frac{B, \theta}{B}\right)=0
$$

Next, from the lowest order in (A7) it follows that in the neighborhood of the center

$$
\Theta_{, \theta} \approx 0 \rightarrow\left(\frac{\dot{C}}{C}\right)_{, \theta} \approx\left(\frac{\dot{B}}{B}\right)_{, \theta} \approx 0,
$$

implying right there

$$
\gamma=f(t) \alpha(\theta), \quad B=g(t) \beta(\theta) .
$$


Then integrating (83) we obtain

$$
H_{1}^{(0)}=\frac{x(t)}{\alpha(\theta)},
$$

where $x(t)$ is an integration function.

An equation derived from the combination of (84) and (87), can also be integrated to produce

$$
H_{2}^{(0)}=\frac{y(t) \beta^{1 / 2}(\theta)}{\alpha^{2}(\theta)}
$$

and a combination of (84) and (87) also produces

$$
2 H_{1}^{(0)}+H_{2}^{(0)} \frac{B, \theta}{B}=0 .
$$

From the equations above it follows that

$$
-2 \frac{x(t)}{y(t)}=\frac{\beta_{, \theta}}{\alpha \beta^{1 / 2}}=\text { constant }
$$

However, this last equation cannot be satisfied. Indeed, because of the reflection symmetry, we have that $\beta(0)=\beta(\pi)$, implying that $\beta_{, \theta}$ must have a change of sign in the interval $[0, \pi]$, whereas $\alpha$ and $\beta$ are positive defined. have:

Thus we must put $H_{1}^{(0)}=H_{2}^{(0)}=0$. Accordingly we

$$
\begin{aligned}
& H_{2}=\sum_{n \geq 1} H_{2}^{(n)} r^{n}, \\
& H_{1}=\sum_{n \geq 1} H_{1}^{(n)} r^{n} .
\end{aligned}
$$

Next, multiplying (A18) by 2 and subtracting from (A14), we obtain at the lowest order of $r$

$$
\mathcal{E}_{1}=\sum_{n \geq 2} \mathcal{E}_{I}^{(n)} r^{n}, \quad \mathcal{E}_{I I}=\sum_{n \geq 2} \mathcal{E}_{I I}^{(n)} r^{n} .
$$

Using the expressions above, we are now looking for the lowest order of $r$ in (A10)-A13) and (A16), we obtain, respectively

$$
\begin{gathered}
H_{1, \theta}^{(1)}=-H_{1}^{(1)} \frac{\gamma_{, \theta}}{\gamma} \\
2 H_{1}^{(1)}-H_{2, \theta}^{(1)}-H_{2}^{(1)}\left(\frac{2 \gamma, \theta}{\gamma}-\frac{B_{, \theta}}{B}\right)=0 \\
2 H_{2}^{(1)}=-H_{1}^{(1)}\left(\frac{\gamma_{, \theta}}{\gamma}-\frac{B_{, \theta}}{B}\right), \\
H_{1, \theta}^{(1)}+H_{1}^{(1)} \frac{B_{, \theta}}{B}-2 H_{2}^{(1)}=0
\end{gathered}
$$

$$
4 H_{1}^{(1)}-H_{2, \theta}^{(1)}-H_{2}^{(1)}\left(\frac{2 \gamma_{, \theta}}{\gamma}+\frac{B, \theta}{B}\right)=0 .
$$

Then, proceeding exactly as we did before, using (89), we are lead to

$$
\frac{\beta, \theta}{\alpha}=\text { constant }
$$

which cannot be satisfied because of the reflection symmetry, as argued before.

Therefore we must put

$$
H_{2}=\sum_{n \geq 2} H_{2}^{(n)} r^{n}
$$

$$
H_{1}=\sum_{n \geq 2} H_{1}^{(n)} r^{n}
$$

At this point we have to stop the procedure followed so far with equations (A10- A13) and (A16), since now the lowest order in $r$ in these equations, contains terms not including $H_{1}$ and $H_{2}$.

Thus let us turn to equations (A6), (A8) (A9).

From the lowest order in (A8) and (A9) we find, respectively

$$
\sigma_{I}^{(1)}=\sigma_{I I}^{(1)}=\frac{p(t)}{\alpha},
$$

and

$$
\Omega^{(1)}=q(t, \theta) \alpha,
$$

with

$$
q_{, \theta}=\frac{2 p(t)}{\alpha^{2}}
$$

where $p(t)$ and $q(t, \theta)$ are arbitrary functions.

Now, by the same arguments based on the reflection symmetry exposed before, it is easily concluded that $p(t)=0$ implying $\sigma_{I}^{(1)}=\sigma_{I I}^{(1)}=0$. Using this result in the lowest order of $r$ in (A6) we obtain $\Omega^{(1)}=0$. We can now feed these results back into (A8) and (A9), and look for the lowest order in $r$. We obtain then that

$$
\sigma_{I I}=\sum_{n \geq 3} \sigma_{I I}^{(n)} r^{n}, \quad \sigma_{I}=\sum_{n \geq 3} \sigma_{I}^{(n)} r^{n} .
$$

Using this last result again in (A6), the lowest order in $r$ now implies $\Omega^{(2)}=0$, which in turn, because of (A2) and (A4) implies

$$
\mathcal{E}_{1}=\sum_{n \geq 3} \mathcal{E}_{I}^{(n)} r^{n}, \quad \mathcal{E}_{I I}=\sum_{n \geq 3} \mathcal{E}_{I I}^{(n)} r^{n} .
$$

Using the results above we can now return to A10(A13) and (A16), since now the lowest order in $r$, in these 
equations, only contains terms with $H_{1}$ and $H_{2}$. Doing so we shall raise the lowest order in $r$ of $H_{1}$ and $H_{2}$, until the moment when the lowest order in these equations contains terms without $H_{1}$ and $H_{2}$. Then we can go again through the whole cycle above. Now, it is a simple matter to see that this procedure may be continued as many times as desired, to obtain that $H_{1}^{(n)}=H_{2}^{(n)}=\mathcal{E}_{I}^{(n)}=$ $\mathcal{E}_{I I}^{(n)}=\sigma_{1}^{n}=\sigma_{I I}^{n}=\Omega^{(n)}=0$ for any value of $n \geq 0$, implying in turn that at the center, these quantities as well as their $r$-derivatives of any order vanish.

Then, assuming that all relevant variables are of class $C^{\omega}$, i.e. that they equal their Taylor series expansion around the center, we can analytically continue the zero value at the center to the whole configuration and therefore, we obtain a conformally flat and shear-free spacetime (i.e. F.R.W.).

\section{CONCLUSIONS}

We have shown that all possible models compatible with the line element (15) and a perfect fluid, are FRW, and accordingly non-radiating (gravitationally). This clearly indicates that, both, the geodesic and the nondissipative conditions, are quite restrictive, when looking for a source of gravitational waves.

Having arrived at this point, the relevant question is: does this result make sense from the physical point of view?

To answer to such a question, let us first remember that, already in the seminal Bondi's paper on gravitational radiation(see section 6 in [10]), it was clearly stated that, not only in the case of dust, but also in the absence of dissipation in a perfect fluid, the system is not expected to radiate (gravitationally) due to the reversibility of the equation of state. The rationale supporting this conjecture is very clear: radiation is an irreversible process, this fact emerges at once if absorption is taken into account and/or Sommerfeld type conditions, which eliminate inward traveling waves, are imposed. Therefore, it is obvious that an entropy generator factor should be present in the description of the source. But such a factor is absent in a perfect fluid, and more so in a collisionless dust. In other words, the irreversibility of the process of emission of gravitational waves, must be reflected in the equation of state through an entropy increasing (dissipative) factor.

In order to delve deeper into this question, let us invoke here the tight relationship between radiation and vorticity mentioned before (see the beginning of Sec. II A).

Now, the equation (A5) in the general (non-geodesic) case (Eq. (B.5) in [1]), reads

$$
\Omega_{, \delta} V^{\delta}+\frac{1}{3}\left(2 \Theta+\sigma_{I}+\sigma_{I I}\right) \Omega+K^{[\mu} L^{\nu]} a_{\mu ; \nu}=0
$$

which of course reduces to A5 if the four acceleration vanishes.

From (A5) it follows at once that if at any given time, the vorticity vanishes, then it vanishes at any other time afterwards. Thus we should not expect gravitational radiation from a physically meaningful system, radiating for a finite period of time (in a given time interval), for otherwise such a radiation will not be accompanied by the presence of vorticity.

But, what happens for the perfect (non-dissipative, non-geodesic) fluid?

In this latter case, the condition of thermal equilibrium (absence of dissipative flux) reads (see eq. (57) in [1])

$$
a_{\mu}=-h_{\mu}^{\beta} \Gamma_{, \beta},
$$

where $\Gamma=\ln T$, and $T$ denotes the temperature.

Feeding back (111) into (110) we get

$$
\Omega_{, \delta} V^{\delta}+\frac{1}{3}\left(2 \Theta+\sigma_{I}+\sigma_{I I}+V^{\mu} \Gamma_{, \mu}\right) \Omega=0 .
$$

Thus, even if the fluid is not geodesic, but is nondissipative, the situation is the same as in the geodesic case, i.e. the vanishing of vorticity at any given time implies its vanishing for any time in the future.

This result is in full agreement with earlier works indicating that vorticity generation is sourced by entropy gradients [20]-25]. At the same time we confirm, by invoking the radiation-vorticity link, the Bondi's conjecture about the absence of radiation for non-dissipative systems.

Finally, two comments are in order before concluding:

- In a recent work [26], the role played by magnetic fields in the generation and survival of vorticity, has been brought out. This strongly suggest that the inclusion of magnetic fields in the discussion of gravitationally radiating sources, deserves further attention.

- Geodesic fluids not belonging to the class considered here (Szekeres) have also been shown not to produce gravitational radiation [27]. This strengthens further the case of the non-radiative character of pure dust distributions.

\section{ACKNOWLEDGMENTS}

L.H. thanks Departament de Física at the Universitat de les Illes Balears, for financial support and hospitality. ADP acknowledges hospitality of the Departament de Física at the Universitat de les Illes Balears. J.O. acknowledges financial support from the Spanish Ministry of Science and Innovation (grant FIS2009-07238). 
Appendix A: Summary of equations for the geodesic case

Below we shall write the equations of the framework developed in [1], for the geodesic and perfect fluid case. Then, equations B.1- B.18 in [1], read, respectively From B.1

$$
\dot{\Theta}+\frac{1}{3} \Theta^{2}+\frac{2}{9}\left(\sigma_{I}^{2}+\sigma_{I} \sigma_{I I}+\sigma_{I I}^{2}\right)+Y_{T}=2 \Omega^{2} .
$$

From B.2

$$
\dot{\sigma}_{I}+\frac{1}{9} \sigma_{I}^{2}+\frac{2}{3} \Theta \sigma_{I}-\frac{2}{9} \sigma_{I I}\left(\sigma_{I}+\sigma_{I I}\right)+Y_{I}=\Omega^{2}
$$

From B.3

$$
\frac{1}{3}\left(\sigma_{I}-\sigma_{I I}\right) \Omega+Y_{K L}=0
$$

From B.4

$$
\dot{\sigma}_{I I}+\frac{1}{9} \sigma_{I I}^{2}+\frac{2}{3} \Theta \sigma_{I I}-\frac{2}{9} \sigma_{I}\left(\sigma_{I}+\sigma_{I I}\right)+Y_{I I}=\Omega^{2} .
$$

From B.5

$$
\dot{\Omega}+\frac{1}{3}\left(2 \Theta+\sigma_{I}+\sigma_{I I}\right) \Omega=0 .
$$

From B.6

$$
\begin{array}{r}
-\frac{1}{\sqrt{B^{2} r^{2}+\tilde{G}^{2}}}\left[\Omega_{, \theta}+\tilde{G} \dot{\Omega}+\Omega\left(\frac{\tilde{G} \dot{C}}{C}+\frac{C_{, \theta}}{C}\right)\right]+\frac{1}{3 B}\left\{2 \Theta^{\prime}-\sigma_{I}^{\prime}-\sigma_{I}\left[\frac{2 C^{\prime}}{C}+\frac{\left(B^{2} r^{2}+\tilde{G}^{2}\right)^{\prime}}{2\left(B^{2} r^{2}+\tilde{G}^{2}\right)}\right]\right. \\
\left.-\sigma_{I I}\left[\frac{C^{\prime}}{C}-\frac{\left(B^{2} r^{2}+\tilde{G}^{2}\right)^{\prime}}{2\left(B^{2} r^{2}+\tilde{G}^{2}\right)}\right]\right\}=0 .
\end{array}
$$

From B.7

$$
\begin{array}{r}
\frac{1}{B}\left(\Omega^{\prime}+\Omega \frac{C^{\prime}}{C}\right)+\frac{1}{3 \sqrt{B^{2} r^{2}+\tilde{G}^{2}}}\left\{\left(2 \Theta-\sigma_{I I}\right)_{, \theta}+\tilde{G}\left(2 \Theta-\sigma_{I I}\right)\right. \\
\left.+\sigma_{I}\left[\frac{B_{, \theta}}{B}-\frac{C_{, \theta}}{C}+\tilde{G}\left(\frac{\dot{B}}{B}-\frac{\dot{C}}{C}\right)\right]-\sigma_{I I}\left[\frac{B_{, \theta}}{B}+\frac{2 C_{, \theta}}{C}+\tilde{G}\left(\frac{\dot{B}}{B}+\frac{2 \dot{C}}{C}\right)\right]\right\}=0 .
\end{array}
$$

From B.8

$$
\begin{aligned}
H_{1}= & -\frac{1}{2 B}\left\{\Omega^{\prime}-\Omega\left[\frac{C^{\prime}}{C}-\frac{\tilde{G} \tilde{G}^{\prime}}{2\left(B^{2} r^{2}+\tilde{G}^{2}\right)}\right]\right\}-\frac{1}{6 \sqrt{B^{2} r^{2}+\tilde{G}^{2}}}\left\{\left(2 \sigma_{I}+\sigma_{I I}\right)_{, \theta}\right. \\
& \left.+\sigma_{I}\left[\frac{B, \theta}{B}+\frac{C_{, \theta}}{C}-\tilde{G}\left(\frac{\dot{B}}{B}-\frac{\dot{C}}{C}\right)\right]-\sigma_{I I}\left[\frac{B, \theta}{B}-\frac{2 C_{, \theta}}{C}+\tilde{G}\left(\frac{2 \dot{B}}{B}-\frac{2 \dot{C}}{C}\right)\right]\right\} .
\end{aligned}
$$

From B.9

$$
\begin{array}{r}
H_{2}=+\frac{1}{6 B}\left\{\left(\sigma_{I}+2 \sigma_{I I}\right)^{\prime}+\sigma_{I}\left[\frac{2 C^{\prime}}{C}-\frac{(B r)(B r)^{\prime}}{B^{2} r^{2}+\tilde{G}^{2}}\right]+\sigma_{I I}\left[\frac{C^{\prime}}{C}+\frac{2(B r)(B r)^{\prime}+3 \tilde{G} \tilde{G}^{\prime}}{2\left(B^{2} r^{2}+\tilde{G}^{2}\right)}\right]\right\} \\
-\frac{1}{2 \sqrt{B^{2} r^{2}+\tilde{G}^{2}}}\left\{\Omega_{, \theta}-\Omega\left[\frac{C_{, \theta}}{C}+\tilde{G}\left(\frac{\dot{B}}{B}+\frac{\dot{C}}{C}\right)\right]\right\} .
\end{array}
$$


From B.10

$$
\begin{array}{r}
\frac{1}{3} \dot{\mathcal{E}}_{I}+\frac{4 \pi}{3}(\mu+P) \sigma_{I}-\Omega \mathcal{E}_{K L}+\frac{\mathcal{E}_{I}}{9}\left(3 \Theta+\sigma_{I I}-\sigma_{I}\right)+\frac{\mathcal{E}_{I I}}{9}\left(2 \sigma_{I I}+\sigma_{I}\right) \\
-\frac{1}{\sqrt{B^{2} r^{2}+\tilde{G}^{2}}}\left(H_{1, \theta}+H_{1} \frac{C_{, \theta}}{C}\right)-\frac{H_{2}}{B}\left[\frac{C^{\prime}}{C}-\frac{2(B r)(B r)^{\prime}+\tilde{G} \tilde{G}^{\prime}}{2\left(B^{2} r^{2}+\tilde{G}^{2}\right)}\right]=0 .
\end{array}
$$

From B.11

$$
\begin{array}{r}
2 \dot{\mathcal{E}}_{K L}+\mathcal{E}_{K L}\left(2 \Theta-\sigma_{I}-\sigma_{I I}\right)+\frac{\Omega}{3}\left(\mathcal{E}_{I}-\mathcal{E}_{I I}\right)+\frac{1}{B}\left\{H_{1}^{\prime}+H_{1}\left[\frac{2 C^{\prime}}{C}-\frac{2(B r)(B r)^{\prime}+\tilde{G} \tilde{G}^{\prime}}{2\left(B^{2} r^{2}+\tilde{G}^{2}\right)}\right]\right\} \\
-\frac{1}{\sqrt{B^{2} r^{2}+\tilde{G}^{2}}}\left\{H_{2, \theta}+H_{2}\left[\frac{2 C_{, \theta}}{C}-\frac{B, \theta}{B}-\tilde{G}\left(\frac{\dot{B}}{B}-\frac{\dot{C}}{C}\right)\right]\right\}=0
\end{array}
$$

From B.12

$$
\begin{aligned}
& \frac{1}{3} \dot{\mathcal{E}}_{I I}+\frac{4 \pi}{3}(\mu+P) \sigma_{I I}+\Omega \mathcal{E}_{K L}+\frac{\mathcal{E}_{I I}}{9}\left(3 \Theta+\sigma_{I}-\sigma_{I I}\right)+\frac{\mathcal{E}_{I}}{9}\left(2 \sigma_{I}+\sigma_{I I}\right) \\
& +\frac{1}{B}\left(H_{2}^{\prime}+H_{2} \frac{C^{\prime}}{C}\right)+\frac{H_{1}}{\sqrt{B^{2} r^{2}+\tilde{G}^{2}}}\left[\frac{C_{, \theta}}{C}-\frac{B, \theta}{B}-\tilde{G}\left(\frac{\dot{B}}{B}-\frac{\dot{C}}{C}\right)\right]=0
\end{aligned}
$$

From B.13

$$
\begin{aligned}
-\frac{1}{3}\left(\mathcal{E}_{I}+\mathcal{E}_{I I}\right)^{\cdot} & -\frac{1}{3}\left(\mathcal{E}_{I}+\mathcal{E}_{I I}\right) \Theta-\frac{4 \pi}{3}(\mu+P)\left(\sigma_{I}+\sigma_{I I}\right)-\frac{\mathcal{E}_{I}}{9}\left(2 \sigma_{I I}+\sigma_{I}\right)-\frac{\mathcal{E}_{I I}}{9}\left(2 \sigma_{I}+\sigma_{I I}\right) \\
& +\frac{1}{\sqrt{B^{2} r^{2}+\tilde{G}^{2}}}\left(H_{1, \theta}+H_{1} \frac{B, \theta}{B}\right)-\frac{1}{B}\left\{H_{2}^{\prime}+H_{2}\left[\frac{(B r)(B r)^{\prime}+\tilde{G} \tilde{G}^{\prime}}{B^{2} r^{2}+\tilde{G}^{2}}\right]\right\}=0
\end{aligned}
$$

From B.14

$$
\begin{array}{r}
\frac{1}{3 B}\left\{\mathcal{E}_{I}^{\prime}+\mathcal{E}_{I}\left[\frac{2 C^{\prime}}{C}+\frac{\left(B^{2} r^{2}+\tilde{G}^{2}\right)^{\prime}}{2\left(B^{2} r^{2}+\tilde{G}^{2}\right)}\right]+\mathcal{E}_{I I}\left[\frac{C^{\prime}}{C}-\frac{\left(B^{2} r^{2}+\tilde{G}^{2}\right)^{\prime}}{2\left(B^{2} r^{2}+\tilde{G}^{2}\right)}\right]\right\} \\
+\frac{1}{\sqrt{B^{2} r^{2}+\tilde{G}^{2}}}\left\{\mathcal{E}_{K L, \theta}+\tilde{G} \dot{\mathcal{E}}_{K L}+\mathcal{E}_{K L}\left[\frac{2 B, \theta}{B}+\frac{C_{, \theta}}{C}+\tilde{G}\left(\frac{2 \dot{B}}{B}+\frac{\dot{C}}{C}\right)\right]\right\} \\
-\frac{1}{3} H_{2}\left(\sigma_{I}+2 \sigma_{I I}\right)-3 \Omega H_{1}=\frac{8 \pi}{3 B} \mu^{\prime} .
\end{array}
$$

From B.15

$$
\begin{aligned}
& \frac{1}{B}\left\{\mathcal{E}_{K L}^{\prime}+\mathcal{E}_{K L}\right. {\left.\left[\frac{C^{\prime}}{C}+\frac{\left(B^{2} r^{2}+\tilde{G}^{2}\right)^{\prime}}{B^{2} r^{2}+\tilde{G}^{2}}\right]\right\}+\frac{1}{3 \sqrt{B^{2} r^{2}+\tilde{G}^{2}}}\left\{\mathcal{E}_{I I, \theta}+\tilde{G} \mathcal{E}_{I I}\right.} \\
&\left.+\mathcal{E}_{I}\left[\frac{C_{, \theta}}{C}-\frac{B_{, \theta}}{B}+\tilde{G}\left(\frac{\dot{C}}{C}-\frac{\dot{B}}{B}\right)\right]+\mathcal{E}_{I I}\left[\frac{2 C_{, \theta}}{C}+\frac{B_{, \theta}}{B}+\tilde{G}\left(\frac{2 \dot{C}}{C}+\frac{\dot{B}}{B}\right)\right]\right\} \\
&+\frac{1}{3} H_{1}\left(2 \sigma_{I}+\sigma_{I I}\right)-3 \Omega H_{2}=\frac{8 \pi}{3 \sqrt{B^{2} r^{2}+\tilde{G}^{2}}}(\tilde{G} \dot{\mu}+\mu, \theta) .
\end{aligned}
$$


From B.16

$$
\begin{array}{r}
\frac{1}{3} \mathcal{E}_{K L}\left(\sigma_{I I}-\sigma_{I}\right)-\frac{1}{B}\left\{H_{1}^{\prime}+H_{1}\left[\frac{2 C^{\prime}}{C}+\frac{\left(B^{2} r^{2}+\tilde{G}^{2}\right)^{\prime}}{2\left(B^{2} r^{2}+\tilde{G}^{2}\right)}\right]\right\} \\
-\frac{1}{\sqrt{B^{2} r^{2}+\tilde{G}^{2}}}\left\{H_{2, \theta}+\tilde{G} \dot{H}_{2}+H_{2}\left[\frac{B, \theta}{B}+\frac{2 C_{, \theta}}{C}+\tilde{G}\left(\frac{\dot{B}}{B}+\frac{2 \dot{C}}{C}\right)\right]\right\}=\left[8 \pi(\mu+P)-\left(\mathcal{E}_{I}+\mathcal{E}_{I I}\right)\right] \Omega .
\end{array}
$$

From B.17

$$
\begin{array}{r}
\frac{1}{3 \sqrt{B^{2} r^{2}+\tilde{G}^{2}}}\left\{\left(\mathcal{E}_{I}+\mathcal{E}_{I I}\right)_{, \theta}+\tilde{G}\left(2 \mathcal{E}_{I I}+\mathcal{E}_{I}\right)^{\cdot}+\mathcal{E}_{I}\left[\frac{C_{, \theta}}{C}+\tilde{G}\left(\frac{\dot{C}}{C}+\frac{B^{2} r^{2}}{B^{2} r^{2}+\tilde{G}^{2}} \frac{\dot{B}}{B}\right)\right]\right. \\
\left.+\mathcal{E}_{I I}\left[\frac{2 C_{, \theta}}{C}+2 \tilde{G}\left(\frac{\dot{C}}{C}+\frac{B^{2} r^{2}}{B^{2} r^{2}+\tilde{G}^{2}} \frac{\dot{B}}{B}\right)\right]\right\}+\frac{\mathcal{E}_{K L}}{B}\left[\frac{C^{\prime}}{C}+\frac{\tilde{G} \tilde{G}^{\prime}}{2\left(B^{2} r^{2}+\tilde{G}^{2}\right)}\right]-\dot{H}_{1} \\
-\frac{1}{3} H_{1}\left(3 \Theta+\sigma_{I I}-\sigma_{I}\right)-H_{2} \Omega=\frac{4 \pi}{3 \sqrt{B^{2} r^{2}+\tilde{G}^{2}}} \mu_{, \theta} .
\end{array}
$$

From B.18

$$
\begin{aligned}
& \frac{1}{3 B}\left\{\left(\mathcal{E}_{I}+\mathcal{E}_{I I}\right)^{\prime}+\mathcal{E}_{I}\left(\frac{2 C^{\prime}}{C}+\frac{\tilde{G} \tilde{G}^{\prime}}{B^{2} r^{2}+\tilde{G}^{2}}\right)+\mathcal{E}_{I I}\left[\frac{C^{\prime}}{C}+\frac{\tilde{G} \tilde{G}^{\prime}}{2\left(B^{2} r^{2}+\tilde{G}^{2}\right)}\right]\right\} \\
& +\frac{1}{\sqrt{B^{2} r^{2}+\tilde{G}^{2}}}\left\{\tilde{G} \dot{\mathcal{E}}_{K L}+\mathcal{E}_{K L}\left[\frac{C_{, \theta}}{C}+\tilde{G}\left(\frac{\dot{B}}{B}+\frac{\dot{C}}{C}\right)\right]\right\}+\dot{H}_{2}+\frac{1}{3} H_{2}\left(3 \Theta+\sigma_{I}-\sigma_{I I}\right)-H_{1} \Omega=\frac{4 \pi}{3 B} \mu^{\prime} .
\end{aligned}
$$

\section{Appendix B: Conformal flatness implied by the vanishing of vorticity}

We shall here provide an alternative proof of the result exhibited in Sec.II about the consequence of assuming vanishing vorticity.

First of all we observe that due to (24), (51) and (61) we may write at $r \approx 0$

$$
\gamma=\frac{L(\theta}{r}, \quad L(\theta) \equiv \frac{1}{l(\theta)}
$$

where $L(\theta)$ must be a regular function of $\theta$.

Then, using (B1) in (63) (always at $r \approx 0$ ) we obtain

$$
H_{2}=-L(\theta) H_{1}
$$

Next, from (A10) and (A12) we obtain

$$
-H_{1, \theta}-H_{1}\left(\frac{2 C_{, \theta}}{C}-\frac{B_{, \theta}}{B}\right)=r\left[H_{2}^{\prime}+H_{2}\left(\frac{2 C^{\prime}}{C}-\frac{(B r)^{\prime}}{B r}\right)\right]
$$

Then, introducing the auxiliary function $b(t, r, \theta)$ defined by

$$
B r=b C^{2}
$$

the equations (B3) and (55) can be rewritten as:

$$
\begin{gathered}
-\left(\frac{H_{1}}{b}\right)_{, \theta}=r\left(\frac{H_{2}}{b}\right)^{\prime}, \\
\left(\frac{H_{2}}{b}\right)_{, \theta}=r\left(\frac{H_{1}}{b}\right)^{\prime},
\end{gathered}
$$

or

$$
\begin{aligned}
& \left(\frac{H_{1}}{b}\right)^{\prime \prime}+\frac{1}{r}\left(\frac{H_{1}}{b}\right)^{\prime}+\frac{1}{r^{2}}\left(\frac{H_{1}}{b}\right)_{, \theta \theta}=0, \\
& \left(\frac{H_{2}}{b}\right)^{\prime \prime}+\frac{1}{r}\left(\frac{H_{2}}{b}\right)^{\prime}+\frac{1}{r^{2}}\left(\frac{H_{2}}{b}\right)_{, \theta \theta}=0 .
\end{aligned}
$$

The equations (B7) and (B8) can be integrated to obtain

$$
\begin{gathered}
H_{1}=b \sum_{n=1}^{\infty} r^{n}\left[\alpha_{n}(t) \cos (n \theta)+\beta_{n}(t) \sin (n \theta)\right], \\
H_{2}=b \sum_{n=1}^{\infty} r^{n}\left[-\beta_{n}(t) \cos (n \theta)+\alpha_{n}(t) \sin (n \theta)\right]
\end{gathered}
$$

where coefficients $\alpha_{n}(t), \beta_{n}(t)$ are arbitrary functions of $t$.

Observe that for $n=0$ we have

$$
H_{1}=\frac{B r}{C^{2}} \alpha_{0}(t), H_{2}=-\frac{B r}{C^{2}} \beta_{0}(t) .
$$


However, in the neighborhood of $r \approx 0$, we have $C \approx r$; implying that $H_{1}$ and $H_{2}$ are singular at the origin, and therefore we must put $\alpha_{0}(t), \beta_{0}(t)=0$.

Next, feeding back (B9) and (B10) into (B3), we have a system of equations for each order of $r^{n}$. Using (B2) we obtain

$$
\alpha_{1}=\beta_{1}=0
$$

where we have explicitly used the fact that $L(\theta)$ is a regular function of $\theta$ (at least on the symmetry axis).

Repeating the calculations for the consecutive orders, it is a simple matter to check that

$$
\alpha_{n}=\beta_{n}=0, \text { for any } \mathrm{n},
$$

thereby proving that $H_{1}=H_{2}=0$. The remaining of the proof follows as in Sec. III.
[1] L. Herrera, A. Di Prisco, J. Ibáñez and J. Ospino, Phys. Rev. D 89, 084034, (2014).

[2] G. F. R. Ellis Relativistic Cosmology in: Proceedings of the International School of Physics "Enrico Fermi", Course 47: General Relativity and Cosmology. Ed. R. K. Sachs (Academic Press, New York and London) (1971).

[3] G. F. R. Ellis and H. van Ellst, gr-qc/9812046v4 (1998).

[4] G. F. R. Ellis Gen. Rel. Grav. 41, 581 (2009).

[5] G. F. R. Ellis, R. Maartens and M. A. H. MacCallum, Relativistic Cosmology (Cambridge U. P., Cambridge) (2012).

[6] L. Herrera, A. Di Prisco and J. Ospino, Phys. Rev. D 89,127502, (2014).

[7] L. Bel, Cah. de Phys. 16, 59 (1962).

[8] A. García-Parrado Gómez Lobo, Class. Quantum Grav. 25, 015006 (2008).

[9] A Di Prisco, L. Herrera, M. A. H. MacCallum and N.O. Santos, Phys. Rev. D 80, 064031 (2009).

[10] H. Bondi, M. G. J. van der Burg and A. W. K. Metzner, Proc. Roy.Soc. A 269, 21 (1962).

[11] L. Herrera, W. Barreto, J. Carot and A. Di Prisco, Class. Quantum Grav., 24, 2645, (2007).

[12] H. Stephani, D. Kramer, M. MacCallum, C. Honselaers, and E. Herlt, Exact Solutions to Einsteins Field Equations (Cambridge University Press, Cambridge, Eng- land), (2003), 2nd Ed.

[13] J. Carot, Class. Quantum Grav. 17, 2675 (2000).

[14] G. T. Carlson, Jr. and J. L. Safko, Ann. Phys. (N.Y.) 128, 131 (1980).

[15] L. Herrera, Gen. Rel. Grav. 46, 1654 (2014).

[16] P. Szekeres, Phys. Rev. D 12, 2941 (1975).

[17] P. Szekeres, Commun. Math. Phys. 41, 55 (1975).

[18] A. Krasinski Inhomogeneous Cosmological Models,(Cambridge University Press, Cambridge) (1998).

[19] C. Hellaby and A. Krasinski, Phys. Rev. D 66, 084011 (2002).

[20] L. Crocco and Z. Agnew, Math. Mech. 17, 1 (1937).

[21] E. R. Harrison, Mon. Not. R. Astron. Soc. 147, 279 (1970).

[22] A. J. Christopherson, K. A. Malik and D. R. Matravers, Phys. Rev. D 79, 123523 (2009).

[23] F. Del Sordo and A. Brandenburg, Astron. Astrophys. 528, A145 (2011).

[24] A. J. Christopherson and K. A. Malik, Class. Quantum Grav. 28, 114004 (2011).

[25] F. Dosopoulou, F. Del Sordo, C. G. Tsagas and A. Brandenburg, Phys. Rev. D 85, 063514 (2012).

[26] F. Dosopoulou and C. G. Tsagas, Phys. Rev. D 89, 103519 (2014).

[27] W. B. Bonnor, Commun. Math. Phys. 51, 191 (1976). 